\title{
Toward Optimal Error Distributions in Adaptive Finite-Element Electromagnetic Analysis for Microelectronic Interconnection Structures
}

\author{
Dennis Giannacopoulos
}

\begin{abstract}
The effectiveness of finite-element refinement criteria for achieving optimal meshes based on error equidistribution principles are investigated with benchmark systems for the electromagnetic simulation of microelectronic system interconnection (MSI) features. The usefulness of the criteria are evaluated for adaptive finite-element electromagnetic analysis of principal device characteristics present in practical MSI structures, which are known to pose challenging problems in numerical modeling. The criteria with, potentially, the most significant implications for MSI electromagnetic simulation, are examined with finite-element solutions for the fundamental benchmark systems computed from both optimal and adaptively refined discretizations.
\end{abstract}

Index Terms-Adaptive systems, electromagnetic analysis, error analysis, finite-element methods (FEMs).

\section{INTRODUCTION}

$\mathbf{R}$ ECENTLY, the study of error distributions corresponding to optimized finite-element discretizations has attracted a good deal of interest in the research community [1]-[3]. An important incentive for this research focus stems from the potential benefits of identifying effective and reliable refinement criteria, for adaptive finite-element analysis (AFEA), based on $a$ priori characterizations of optimal discretization solution properties. Such refinement criteria could be used in practice for efficiently guiding adaptive finite-element electromagnetic solvers toward optimal accuracy solutions, without incurring the prohibitive computational costs associated with solving the optimal discretization problem directly [2]. This may be especially important for overcoming the computational bottleneck which is, currently, a key obstacle for practical finite-element electromagnetic analysis of realistic MSI structures [4].

The main difficulty with computational MSI analysis is that a very large number of free modeling parameters are needed to compute accurate and reliable simulations for realistic systems. Sufficient mathematical degrees-of-freedom (DOF) are required to both resolve the geometric and material features of a MSI structure, and represent the fields of the electromagnetic system. The computational effort required for the electromagnetic analysis of the complex, dense, and irregularly routed arrays of high-speed interconnections that comprise modern MSI structures can often be prohibitive [4]. Yet such analyzes are

Manuscript received July 5, 2001; revised October 25, 2001. This work was supported in part by the Natural Sciences and Engineering Research Council of Canada.

The author is with the Department of Electrical and Computer Engineering, McGill University, Montreal, QC H3A 2A7, Canada.

Publisher Item Identifier S 0018-9464(02)00895-6. critical if MSI system performance is to be simulated with confidence.

In recent years, significant advances have been made on the electromagnetic modeling, simulation and computer-aided design of MSI structures [5]. Currently, one promising way to overcome this type of computational barrier is by using adaptive solver technologies which are capable of intelligently evolving and improving an efficient distribution of DOF over the problem domain [4]. Such methods begin with relatively inexpensive initial discretizations, then establish operational solution error distributions over them, and subsequently add DOF to the model to correct them [2]. However, the potential additional benefits and implications of achieving optimal error distributions in AFEA for MSI simulation have yet to be reported in the mainstream literature, and are the focus of this work.

Some of the most prominent and enduring contributions to emerge in the literature on optimal error distributions for numerical methods have been based on the so-called equidistribution principle (EP) [6]-[8]. According to this principle, the ideal mesh for a given number of DOF, i.e., the mesh that produces the most accurate solution, will exhibit a homogeneous element-wise error distribution over the problem domain. However, incidental results from recent studies indicate that the EP may not be a universally valid mesh optimality criterion for electromagnetic AFEA [1]. In this work, it is shown directly for the first time that the EP does not, in general, lead to optimal error distributions. The implications of these findings are especially significant for electromagnetic AFEA, since many feedback control systems used to guide the adaptive refinement process are currently based on the EP. Moreover, it should be noted that the experimental evidence supporting the conclusions presented in this study, are based on error data computed from optimal finite-element discretizations. Thus, the results computed under these conditions can not be refuted. In contrast, previous studies published on typical, or nonoptimal meshes, have not provided the sufficiently rigorous and conclusive experimental evidence required for examining the fundamental value of the EP. Therefore, the novelty of this work is, in part, the use of optimally discretized benchmark systems for evaluating the validity of the EP: a critical component in obtaining the definitive evidence required. The purpose of this contribution is to investigate the practical value of both previously established and newly proposed error EPs for the electromagnetic AFEA of key MSI features, in order to better understand the true impact and merit of the concepts for overcoming the significant inherent computational barriers. 


\section{EQUIDISTRIBUTION PRINCIPLES}

The EP has long held a prominent place in the design of feedback control systems used to guide the refinement process for updating discretizations in AFEA [6]-[8]. Its enduring popularity has been attributed to the simplicity and strength of the underlying fundamental concept [8]: refining a discretization based on equidistribution of the solution error over all of the elements in a mesh, will yield the optimal mesh for a given problem. Thus, the EP prescribes a conceptually intuitive and natural approach for determining how to update a discretization in order to produce the most accurate solution for a given number of DOF [9]. Consequently, due to its generality and potentiality, the EP has been used with a range of finite-element refinement criteria based on various measures of the solution error [8]. In most cases, AFEA based on the EP has been shown to result in good error convergence performance relative to nonadaptive uniform refinement approaches. However, in the following sections it is demonstrated directly that the EP does not, in general, correspond to optimal error distributions for finite-element electromagnetics. Subsequently, a new EP is proposed based on refinement criteria that are derived expressly from the optimal discretization principle itself, and its practical value is examined for AFEA.

\section{A. Previously Established EPs}

One of the most general and powerful formulations for an EP was given in [6], and introduced the concept of the grading function. By definition, a grading function is a function whose value changes by a constant amount over each element in a discretization. Mathematically, a grading function is a convenient means by which to describe, or characterize, the placement of element vertices in a finite-element mesh. For example, in a one-dimensional (1-D) mesh with $N$ elements, a grading function $\xi(x)$ must satisfy the following:

$$
\xi\left(x_{i}\right)-\xi\left(x_{i-1}\right)=\int_{\Omega_{i}} \xi^{\prime} d x=\frac{1}{N}
$$

where $x_{i-1}$ and $x_{i}$ are the coordinates of adjacent element vertices defining the $i$ th element over the subregion $\Omega_{i}$ of the discretized problem domain. The general approach taken in [6] is to derive such a grading function that will minimize the approximation error in a computed finite-element solution for a given problem. In other words, the optimal discretization will be that which has element vertices positioned such that (1) is satisfied for the derived grading function. More specifically, the grading function derived in [6] is given by

$$
\xi(x)=\frac{\int_{a}^{x}\left[u^{(k+1)}\right]^{2 /[2(k+1-m)+1]} d x}{\int_{a}^{b}\left[u^{(k+1)}\right]^{2 /[2(k+1-m)+1]} d x}
$$

where: the problem domain is bounded by the interval $[a, b] ; k$ represents the polynomial order of the approximate finite-element solution; $u$ is either the known analytical field solution or an extrapolation of the approximate solution of at least order $k+1$; and the approximation error is measured in terms of the $H^{m}$-seminorm. In order to examine the effectiveness of the EP based on this grading function approach, the $H^{1}$-seminorm form is employed, which is consistent with the variational finite-element formulation used in this work [6]. Namely, this EP is analyzed in the following section using a series optimal discretization results computed for a basic benchmark system.

A second type of error EP based on the residual of the governing partial differential equation (PDE) of the physical system, is also considered in this work [8]. Specifically, the equidistribution of the element-wise PDE residual:

$$
\left[\int_{\Omega_{i}}\left(\nabla^{2} \tilde{u}_{i}\right)^{2} d \Omega\right]^{1 / 2}
$$

is analyzed for a free-space Laplace benchmark system in the following section, where $\tilde{u}_{i}$ represents the approximate finiteelement solution for the $i$ th element over the sub-region $\Omega_{i}$ of the discretized problem domain. Unlike the grading function EP discussed above, equidistributing the residual of the PDE has not been justified with rigorous theoretical analyzes, but rather, has most often been employed heuristically. One of the most positive attributes of PDE residual based refinement criteria is the direct measure they can provide of how well the computed solutions satisfy the differential equations used to mathematically model a physical system. This intuitive and simple approach is often more readily accepted by finite-element engineering communities than more mathematically abstract optimality criteria, for example, those based on the grading function approach described above. Therefore, PDE residual-based refinement criteria have been developed and investigated extensively for electromagnetic AFEA [8] and are also considered here. The third type of error EP examined, is based on the simple modification of scaling the PDE residual (3) over an element by the element size.

\section{B. Newly Proposed EP}

One approach that may prove successful for ensuring an EP will yield optimal error distributions, is to employ refinement criteria that are inherently derived from an explicit formulation for computing optimal finite-element discretizations directly. Such a formulation has been derived in [10] based on variational methods for general scalar Helmholtz systems. Namely, in [10] a set of optimization equations are defined for the geometric discretization parameters (i.e., the element vertex positions), which can be used to define AFEA refinement criteria. Specifically, the relative discretization errors over a nonoptimal finite-element mesh can be estimated in terms of how well these equations are satisfied by that discretization. These optimization equations are defined element-wise, with respect to vertex positions, and they can be evaluated locally to indicate the relative optimality of the elements in a mesh. Hence, the optimal discretization based refinement criteria are defined implicitly as measures of the residuals of the geometric optimization equations, which will evaluate identically to zero for an optimal mesh [10]. Therefore, the newly proposed EP is based precisely on equidistributing these residuals over each element in a discretization. 


\section{RESULTS}

A simple 1-D free-space example is presented in order to examine the effectiveness of the three previously established EPs described above for achieving optimal finite-element error distributions. In addition, the practical significance of using the newly proposed EP is examined, for a range of AFEA models, with a 2-D Laplace system incorporating principal device features of practical MSI structures.

\section{A. 1-D: Free-Space Point Charge Test System}

This static benchmark system was first studied in [11], and is based on the classical point singularity model in free-space. The objective for this benchmark system is to compute the functional value based on the resolution of a radial neighborhood close to the point charge and spanning a 100-fold decay in electric scalar potential: the point charge, of magnitude $10^{-9} / 9 \mathrm{C}$, is located at the origin, and the two boundaries of the problem domain are set at radial distances of $0.1 \mathrm{~m}$ and $10 \mathrm{~m}$ away from the charge. It may be noted, that the field solution associated with the free-space point charge model contains the type of singularity associated with the sharp material corners which are present in many practical MSI structures [4]. Thus, the primary feature of this system is the rapid field solution variation close to the singularity, and has been shown to drastically reduce the finite-element convergence rate.

The effectiveness of the three previously established error EPs described in the last section are considered for this example. All results were computed using standard Lagrangian elements for the analysis of this 1-D example, and are based on solving the optimization equations for the geometric discretization parameters [10]. Therefore, each of the optimal discretization results computed for this benchmark system represents the ideal mesh for a given number of DOF, i.e., the mesh that produces the most accurate solution possible for the variational finite-element formulation used [10]. Consequently, the effectiveness of the error EPs considered, are evaluated directly with respect to these optimal finite-element solutions.

The average percent errors in the grading function EP computed for optimal second-order discretizations for the 1-D freespace test system are shown in Fig. 1. The percent errors were calculated based on satisfying the fundamental EP of (1), where the $H^{1}$-seminorm form of the grading function (2) was analyzed using optimal discretization results computed for this benchmark system. Hence, for each of the optimal second-order solutions considered, the average percent error was calculated as the mean of the percent error in satisfying this basic definition over each individual element, and is seen lie in the range from approximately $22 \%$ to $41 \%$. It should be noted that the general form of the grading function (2) will, by definition, change by an average amount of $1 / N$ over each element in any $N$-element mesh; however, for an optimal $N$-element mesh the grading function should change by the exact amount of $1 / N$ over each element, which is clearly not the case for this system.

The average percent errors in equidistributing the PDE residual computed for the optimal second-order discretizations for this benchmark system are also shown in Fig. 1. For each of the optimal second-order solutions considered, the average

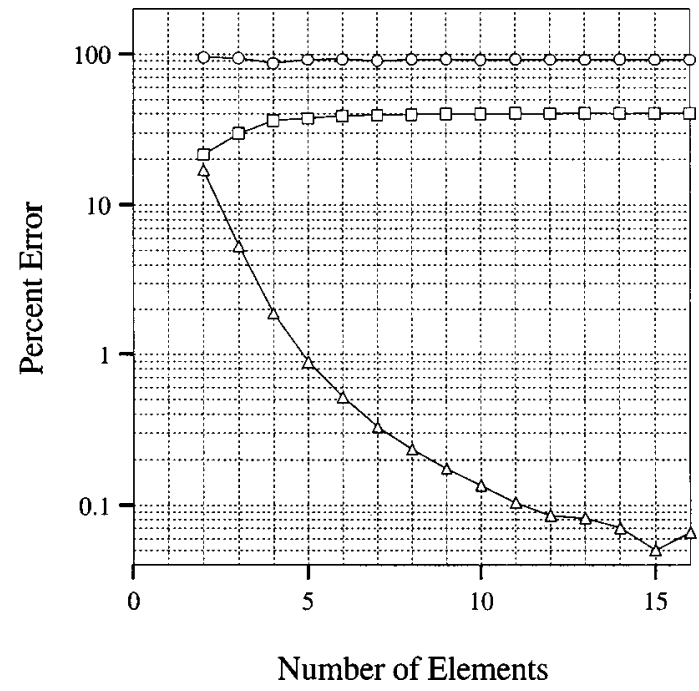

Fig. 1. Equidistribution error versus number of elements for optimal second-order discretizations of the 1-D free-space test system. The square-, circle-, and triangle-knot results correspond to the grading function, the PDE residual and the modified PDE residual EPs, respectively.

percent error in the PDE residual was calculated as the mean of the percent error in equidistributing the PDE residual over each individual element, and lies in the range from approximately 90-96\%. When the modified PDE residual (based on scaling by the element size) was evaluated for the same optimal discretization-based solutions, it was observed that, overall, the error in equidistributing this modified residual was significantly reduced compared to the unscaled version considered above. Fig. 1 shows that the average error in equidistributing the PDE residual scaled by the element size over each element in a mesh smoothly converged from $17 \%$ down to $0.05 \%$ for meshes ranging from 2 to 16 elements.

The relatively high percent errors reported above for the EPs evaluated for the 1-D free-space test system, confirm that they do not correspond to optimal error distributions for electrostatic finite-element solutions. Furthermore, for each of the EPs considered for this benchmark system, analogous first-, fourth-, and eighth-order results were computed that corroborate the second-order results presented. Although, the principle of equidistributing the PDE residual scaled by the element size appears to be more effective for characterizing optimal finite-element discretizations for the benchmark system considered, it would not be prudent to recommend this modified principle without providing theoretical justification, and without further investigating its effectiveness for a wider range of problems. Finally, it must be noted that the newly proposed EP, described in the previous section, is by definition satisfied identically for each of the optimal discretization solutions computed; therefore, the practical value of this new EP is studied next.

\section{B. 2-D: Multipath Transmission Structure}

A 2-D test system is examined to illustrate the practical value of the newly proposed optimal discretization based EP for electromagnetic AFEA. The test system is a translational multi-path transmission structure consisting of three rectangular stripline 


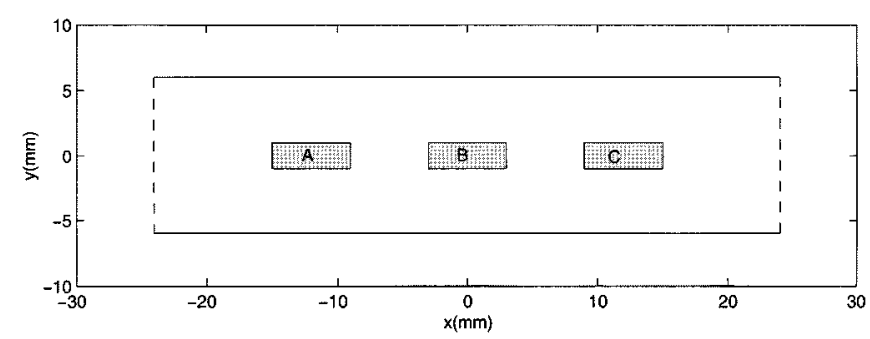

Fig. 2. Geometry for the multipath transmission structure.

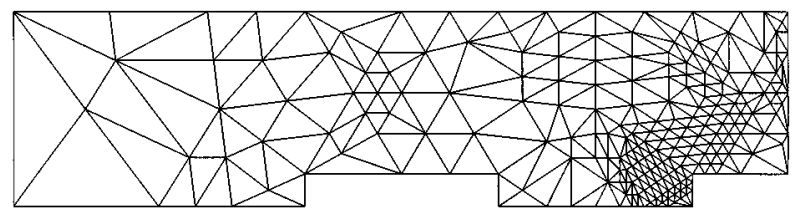

Fig. 3. An example of an $h$-adapted mesh (upper left quarter).

TABLE I

Discretization LeVEL VS. PERCENT ERROR IN ENERGY

\begin{tabular}{ccccc}
\hline Method / \#DOF & $\mathbf{5 . 0 \%}$ & $2.5 \%$ & $\mathbf{1 . 0 \%}$ & $0.5 \%$ \\
\hline Uniform $h$-refinement & 140 & 415 & 3102 & 4752 \\
Adaptive $h$-refinement & 101 & 179 & 418 & 650 \\
Uniform $p$-refinement & 145 & 342 & 483 & 817 \\
Adaptive $p$-refinement & 145 & 229 & 288 & 512 \\
Adaptive $h p$-refinement & 140 & 220 & 300 & 383 \\
\hline
\end{tabular}

conductors, buried within a dielectric substrate, between two solid conductor planes [12]. A crosssectional view of the geometry is provided in Fig. 2. The relative permittivity of the substrate is 2.2 , and the dashed vertical lines represent symmetry planes in the truncated model. This type of MSI structure is common in multichip module (MCM) technology [12]; the primary feature is the highly nonuniform variation of the field solution over the problem domain. The new EP can be used to guide AFEA toward optimal meshes, by iteratively refining the discretizations based on equidistributing the residuals of the optimization equations for the geometric discretization parameters [10]. For the purpose of the following tests, the striplines labeled $\mathrm{A}$ and $\mathrm{C}$ were each set to a potential of $25 \mathrm{mV}$ higher than the two reference planes; and stripline $\mathrm{B}$ was set to a potential of $100 \mathrm{mV}$ above the reference planes. The objective of the analyzes was to resolve the electrostatic energy per unit length.

The performance results for $h-, p$ - and $h p$-adaption strategies, based on the new EP, are reported in Table I. The uniform $h$ and $p$-refinement baseline result are included for comparison. The $h$-adaption results reported are for first-order triangles, and $50 \%$ increments in the number of DOF were used to refine the discretization at each adaptive step; the $p$ - and $h p$-discretizations ranged over orders 1 through 5, and a $100 \%$ increment in the number of DOF per adaptive step was used to improve the discretizations (excluding the uniform refinement procedure). In each case, compared to the uniform refinement baselines, a

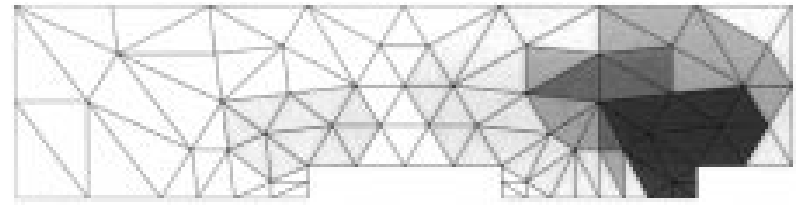

Fig. 4. An example of a $p$-adapted mesh (upper left quarter).

considerable savings in the number of DOF was achieved using the new EP for all of the solution accuracy levels reported. Example $h$ - and $p$-adapted meshes are presented in Figs. 3 and 4, respectively, to illustrate the sharp focus of DOF produced by the new EP. The shading intensity in Fig. 4 represents the element orders: white $=1$; dark grey $=5$.

\section{CONCLUSION}

In this paper, previously established EPs were shown, in general, not to correspond to optimal error distributions for finite-element electromagnetic solutions. Moreover, a newly proposed $\mathrm{EP}$, based on refinement criteria derived expressly from the optimal discretization principle itself, was examined for practical AFEA of the electromagnetic behavior of key MSI device features. The new EP was shown to result in improved solution error convergence compared to uniform refinement procedures.

\section{REFERENCES}

[1] L. Janicke, A. Kost, and P. A. Bastos, "Local error distribution in adaptively generated meshes," IEEE Trans. Magn., vol. 36, pp. 1627-1630, July 2000.

[2] S. McFee and D. Giannacopoulos, "Optimal discretization based refinement criteria for finite-element adaption," IEEE Trans. Magn., vol. 32, pp. 1357-1360, May 1996.

[3] L. Y. Li and P. Bettess, "Notes on mesh optimal criteria in adaptive finiteelement computations," Com. Num. Meth. Eng., vol. 11, pp. 911-915, 1995.

[4] S. McFee and D. Giannacopoulos, "The implications of adaptive finite-element analysis on electromagnetic simulation for microelectronic system interconnections," in IEEE Proc. 42nd Midwest Symp. Circuits Systems, 2000, pp. 325-332.

[5] A. Polycarpou, P. Tirkas, and C. Balanis, "The finite-element method for modeling circuits \& interconnects for electronic packaging," IEEE Trans. Microwave Theory Tech., vol. 45, pp. 1868-1874, Oct. 1997.

[6] G. Carey and H. Dinh, "Grading functions and mesh redistribution," SIAM J. Num. Anal., vol. 22, no. 5, pp. 1028-1040, 1985.

[7] K. E. Chen, "Error equidistribution and mesh adaptation," SIAM J. Sci. Comp., vol. 15, no. 4, pp. 798-818, 1994.

[8] M. Salazar-Palma, T. K. Sarkar, L. E. Garcia-Castillo, T. Roy, and A. Djordjevic, Iterative and Self-Adaptive Finite-Elements in Electromagnetic Modeling. Boston, MA: Artech, 1998.

[9] L. Janicke and A. Kost, "Choosing refinement percentage in adaptive mesh generation," in Digest 9th Biennial IEEE Conf. Electromagnetic Field Computation, 2000, p. 184.

[10] D. Giannacopoulos, "Optimal discretization-based adaptive finite-element analysis for electromagnetics," Ph.D. dissertation, McGill University, Montreal, Canada, 1998.

[11] D. Giannacopoulos and S. McFee, "Toward optimal $h-p$ adaption near singularities in finite-element electromagnetics," IEEE Trans. Magn., vol. 30, pp. 3523-3526, Sept. 1994.

[12] B. K. Gilbert and G. W. Pan, "MCM packaging for present- and nextgeneration high clock-rate digital- and mixed-signal electronic systems: Areas for development," IEEE Trans. Microwave Theory Tech., vol. 45, pp. 1819-1835, Oct. 1997. 